\title{
Intracellular drug uptake - a comparison of single cell measurements using ToF-SIMS imaging and quantification from cell populations with LC-MS/MS
}

\author{
Carla F. Newman ${ }^{*},{ }^{\ddagger}$, Rasmus Havelund ${ }^{\ddagger}$, Melissa K. Passarelli ${ }^{\star}$, Peter S. Marshall ${ }^{\dagger}$, Ian Francis ${ }^{\dagger}$, \\ Andy West ${ }^{\dagger}$, Morgan R. Alexander ${ }^{¥}$, Ian S. Gilmore** and Colin T. Dollery ${ }^{\dagger}$ \\ ${ }^{\dagger}$ GlaxoSmithKline, Stevenage, United Kingdom \\ * National Centre of Excellence in Mass Spectrometry Imaging (NiCE-MSI), National Physical laboratory (NPL), \\ Teddington, Middlesex, United Kingdom \\ ${ }^{¥}$ Division of Advanced Materials and Healthcare Technologies, School of Pharmacy, University of Nottingham, University \\ Park, Nottingham, United Kingdom
}

\begin{abstract}
ToF-SIMS is a label free imaging method that has been shown to enable imaging of amiodarone in single rat macrophage (NR8383) cells. In this study, we show the method extends to three other cell lines relevant to drug discovery: Human embryonic kidney (HEK293), cervical cancer (HeLa) and liver cancer (HepG2). There is significant interest in the variation of drug uptake at the single cell level and we use ToF-SIMS to show that there is great diversity between individual cells and when comparing each of the cell types. These single cell measurements are compared to quantitative measurements of cell-associated amiodarone for the population using LC-MS/MS and cell counting with flow cytometry. NR8383 and HepG2 cells uptake the greatest amount of amiodarone with on average 2.38 and 2.60pg per cell respectively, and HeLa and Hek 293 have a significantly lower amount of amiodarone at 0.43 and $0.36 \mathrm{pg}$ per cell respectively. The amount of cell-associated drug for the ensemble population measurement (LC-MS/MS) is compared with the ToF-SIMS single cell data: similar amount of drug was detected per cell for the NR8383, and HepG2 cells at a greater level that for the HEK293 cells. However, the two techniques did not agree for the HeLa cells and we postulate potential reasons for this.
\end{abstract}

Since the beginning of the last century the pharmaceutical industry has transformed people's lives, creating medicines that either cure diseases or in the worst-case scenario transform diseases that were regarded as terminal into chronic conditions. However, the development of new drugs is becoming increasingly challenging as a result of several factors including: increased target complexity, changing market demands, diminishing returns and ever-stricter safety requirements ${ }^{1}$. A paradigm shift in the drug discovery workflow is required to reduce new candidate attrition and transform classic drug screening assays into analytical platforms that allow better understanding of the compound's behavior, effect and distribution in-vitro, ex-vivo and ideally in-vivo for both animal models and human patients.

Drug discovery faces a struggle with reliable animal models that are effectively representative of the drug's influence on the patient, hence its absolute reliance on clinical trials. Human clinical trials are always going to be a fundamental part of the drug development process, as only the human body can convey its complexity ${ }^{2,3}$.

There are several steps that precede human clinical trials that steer the direction of the drug discovery and development process. Generally, the drug discovery process within a disease area starts with identifying a potential target, by conducting experiments centered on the biology of the disease target, an endogenous entity responsible for the disease state in question (target identification). Typically, this is followed by a High Throughput Screening (HTS) campaign to identify molecules that have an effect on the target of interest (the discovery phase). This needs to be done in a time effective manner as pharmaceutical compound libraries are extensive ${ }^{4,5}$. It is extremely challenging to design a screening campaign that has the necessary speed to cover sufficient candidate compounds in a biological system that is fully representative of the disease model studied. Hence, there is need to develop low throughput assays that follow these high throughput efforts to interrogate the mechanism behind the drug's effect observed in the HTS and validate the compound activity in-vivo (lead validation) ${ }^{6-8}$. These assays are often cell and animal based. However, both the cell and the animal are like black boxes in that there is typically no detailed knowledge of the intricate complexity of the target's biology and decisions are made based on phenotypic responses. ${ }^{9,10}$

Too often the results of these efforts are negative and the project fails to find a suitable drug candidate. This can be for a number of reasons such as poor cell membrane permeability of the compounds, off target activity and inaccessibility of the target ${ }^{6}$. A better understanding of the reasons why the compound failed needs to be established, which requires the ability to see into the black box so the compound can be re-designed ${ }^{7}$.

There are several techniques that are used currently to probe the biology of the disease target within a single cell ${ }^{11}$, all with strong points and limitations. For instance, MALDI (Matrix Assisted Laser Desorption Ionisation) has shown great results in characterizing the distribution of drug compounds in tissues ${ }^{12}$. However, it has traditionally had poor spatial resolution and, limiting its suitability for interrogating cells in culture, as it cannot resolve the sub-cellular distribution. Recent advances have improved the spatial resolution to below $5 \mu \mathrm{m}$ allowing individual cells to be resolved, but sub-cellular structure are yet to be published ${ }^{13,14}$. Other disadvantages of MALDI are the requirement for a matrix and the fact that it does not produce three dimensional information, as the matrix extracts the analyte to the surface of the sample practically limiting 3D imaging to reconstruction from the analysis of sequential tissue sections.

In contrast, electron and super-resolution light microscopy can resolve sub-cellular features. Recently, Chéreau, et al use STED 
(stimulated emission depletion) microscopy to study morphological interactions between astrocytes and synapses with an approximate resolution of $20 \mathrm{~nm}$ per pixel ${ }^{15}$. This was achieved with the use of fluorescent tags and similarly a pharmaceutical compound could be labeled in order to determine its intracellular localization. However, this process will affect the physical-chemical properties of the pharmaceutical as fluorescent tags tend to be similar in molecular weight to the vast majority of small molecule pharmaceutical compounds ${ }^{16-19}$. LC-MS/MS can also be used to determine the presence of drug inside the cell ${ }^{20}$ as it is very sensitive and can be quantitative; however it provides no localization information since the cells must be lysed to extract the analyte. In summary, current technology is limited to either label-free at micron resolution or sub-micron resolution with the addition of tags. ToF-SIMS can bridge the gap as it allows compound identification based on molecular mass and has good spatial resolution. Previously ${ }^{21}$, we demonstrated this in a macrophage cell line (NR8383) where amiodarone was identified and localized in 3D with a resolution of $3.4 \mu \mathrm{m}$ for the intact drug molecule and $500 \mathrm{~nm}$ when using the more intense iodine signal (a characteristic element in the drug).

This cellular model was chosen for a proof of concept study because there is ample evidence in the literature that the NR8383 cell line accumulates amiodarone rather than metabolize it, which leads to an increase in size due to the accumulation of compound and disruption of lipid metabolism ${ }^{22-24}$.

Amiodarone is an anti-arrhythmic drug; its mode of action is to delay the re-polarization of cardiac smooth muscle cells which translates into a steadier heartbeat, however, despite its effectiveness, it causes serious side effects and is therefore only prescribed to patients as a last resort ${ }^{25}$. Amiodarone is known to accumulate inside macrophages causing phospholipidosis (PLD) which under a light microscope gives rise to what appear to be "holes" in the cytoplasmatic region; this is known in microscopy as a "foamy" cell ${ }^{24,26,27}$. However, this phenotypic change does not confirm the presence of compound, only drug induced morphological changes. It is not known if the compound undergoes metabolic degradation whilst in the lysosome, or if exocytosis of the compound occurs as the cells try to cope with the compound accumulation ${ }^{28}$. Furthermore, amiodarone has a high $\log \mathrm{P}$ value (7.64) and therefore has good ToF-SIMS sensitivity ${ }^{29}$.

To the best of our knowledge, our previous publication ${ }^{21}$ was the first time unlabelled drug was imaged in a single mammalian cell using ToF-SIMS at physiological levels. For drug discovery and development, it is important to evaluate the applicability to a wider range of compounds and to other cell types that are commonly used in drug discovery. Therefore, here we investigate the applicability of ToF-SIMS to image uptake of amiodarone into Hek293, HeLa and HepG2 cells.

The Hek293 cell line is commonly used in drug discovery research and so is arguably of wider relevance than NR8383. In addition, the Hek293 cell morphology does not change much compared to the more mobile NR8383, whose role as macrophages is phagocytosis ${ }^{30-32}$. HeLa cells are the most used cell line in research, for example, GSK use HeLa cells in an assay developed to measure intracellular compound concentration $^{33}$. HepG2 cells have been extensively used for research as they are an acceptable hepatocyte model, these cells are very easy to grow, and display epithelial properties, therefore are more manageable than hepatocytes ${ }^{34}$.

Hek293 and HeLa were chosen for this study not only because they are commonly used in drug discovery, but also because we do not expect amiodarone to have a distinct biological effect as seen in the NR8383 cell line. For HepG2 there is evidence of amiodarone playing a role in phospholipidosis. ${ }^{35}$

ToF-SIMS is an ultra-high vacuum technique that makes use of two beams: the analysis beam and the sputter beam. Primary ions collide with the sample surface and through a process known as sputtering, secondary ions from the uppermost surface (1-2 nm) are emitted and subsequently analysed in a mass spectrometer. ToF-SIMS allows 3D imaging of the chemical composition, using, typically, $\mathrm{Bi}_{3}{ }^{+}$focused ion beam for analysis with an $\mathrm{Ar}_{2000}{ }^{+}$sputter beam to erode the sample surface without causing sub-surface molecular damage ${ }^{36}$.

As the majority of drug targets are intracellular ${ }^{3}$ several promising analytical techniques are being developed to study drug uptake and target engagement within cells ${ }^{37}$. The ability to visualize unlabelled compounds inside the cell at physiological dosages can offer valuable insight on the compound's behavior both on and off-target. In this study, we show evidence of an unlabelled pharmaceutical, amiodarone, inside four different types of mammalian cell lines using ToF-SIMS analysis. In parallel, Liquid Chromatography - Tandem Mass Spectrometry (LC-MS/MS) was used to quantify the cell associated drug concentration, resulting in a semi-quantitative imaging approach. We have previously shown that ToF-SIMS can determine if a compound is inside the cell and outside nuclear regions $^{23}$. However, the ToF-SIMS data is not readily quantitative, since the secondary ion intensities are prone to modification by matrix effects ${ }^{34,38}$ Here, we compare the amount of cell-associated amiodarone in individual (ToFSIMS) with the average population (LC-MS/MS) and investigate whether the ToF-SIMS intensity can be calibrated using LC-MS/MS, a well validated quantitative technique, to determine the amount of drug in cells ${ }^{20}$.

\section{EXPERIMENTAL METHODS}

Sample preparation. HepG2, (ATCC HB-8065, Manassas, VA), Hek293 (ATCC CRL-1573, Manassas, VA) and HeLa (ATCC CCL-2, Manassas, VA) cell lines were grown in Eagle's Minimum Essential Medium with $10 \%$ dialyzed fetal bovine serum (Gibco, USA) for at least 4 passages. NR8383 (ATCC CRL-2192, Manassas, VA) were grown in Ham's F-12K Nutrient Mixture (Gibco, USA) supplemented with $15 \%$ dialyzed fetal bovine serum (Gibco, USA) also for at least 4 passages. All cell lines were then cultured in a Nunc Lab-Tek II Chamber slide system (ThermoFisher Scientific, USA) at a density of $0.5 \times 10^{5}$ cells per $\mathrm{mL}$, which was measured using a Vi-Cell-XR (Beckman Coulter, USA). All cell lines were left to settle for 2 hours and extra media was added so the final concentrations of amiodarone were: $1.2,2.4,4.8$ and 9.7 $\mathrm{nmol} / \mathrm{mL}$ amiodarone hydrochloride (Sigma-Aldrich, CAS $19774-82-4$, and purity $\geq 98 \%$ ). The cells were left to incubate for either 2 or 72 hours at $37^{\circ} \mathrm{C}$ and $5 \% \mathrm{CO}_{2}$. They were then 
washed 3 times with $150 \mathrm{mM}$ ammonium formate (SigmaAldrich, USA) $\mathrm{pH} 7.4$.

The samples used for ToF-SIMS were freeze dried on the labtek glass substrate using a CoolSink XT 96F plate (BioCision, USA) under vacuum in a adVantage wizard 2.0 bench top freeze dryer (VirTis, USA) for approximately 1 hour, after this they were stored at negative $80^{\circ} \mathrm{C}$ until analysis. Having been brought to room temperature in a vacuum chamber the slides were inserted into the ToF-SIMS V. (ION-TOF, Munster, Germany)

ToF-SIMS. Experiments were conducted using a TOF.SIMS 5 mass spectrometer (ION-TOF, Munster, Germany) in a dualbeam setup with an electron gun used for charge compensation. The instrument was operated in two modes: (1) High-resolution imaging mode using $\mathrm{a} \mathrm{Bi}_{3}{ }^{2+}$ analysis beam at $60 \mathrm{keV}$, and an $\mathrm{Ar}_{3000}{ }^{+}$sputtering beam at $10 \mathrm{keV}$ in a non-interlaced mode. The primary ion beam was in the "Fast Imaging" mode with an approximate spatial resolution of $0.25 \mu \mathrm{m}$. Pulsed delayed extraction of secondary ions was employed for improved mass resolving power. The $3 \mathrm{D}$ images were collected in positive ion mode over a $150 \mu \mathrm{m} \times 150 \mu \mathrm{m}$ surface area and $256 \times 256$ pixels. In each analysis phase, the $\mathrm{Bi}_{3}{ }^{2+}$ ion dose was $5.5 \times 10^{10}$ ions $/ \mathrm{cm}^{2}$, and an $\mathrm{Ar}_{3000}{ }^{+}$dose of $8.99 \times 10^{13}$ ions $/ \mathrm{cm}^{2}$ was used to erode the cells between analysis phases. The cells were fully eroded after a total of 50 analysis-sputter cycles for the images presented in this publication. (2) Low-resolution "Spectrometry" mode was used with a higher primary ion beam current to increase the number of cells that could be analysed (albeit with poorer but sufficient spatial resolution). $\mathrm{A} \mathrm{Bi}_{3}{ }^{+}$ analysis beam set at $30 \mathrm{keV}$ and an $\mathrm{Ar}_{3000}{ }^{+}$sputtering beam at $10 \mathrm{keV}$ were used for a total of 20 scans. The $\mathrm{Ar}_{3000}{ }^{+}$ion dose in each sputter phase was twice of that above so that the total $\mathrm{Ar}_{3000}{ }^{+}$ion dose was similar between the two experiments. The data analysis was performed in SurfaceLab (ION-TOF, Munster, Germany) (version 6.5). The data processing for the low-resolution mode experiment was performed by defining each individual cell as a region of interest and processing the dataset to obtain the total amiodarone molecular ion intensity.

LC-MS/MS. Cells were grown using T175 flasks (Corning, USA) and seeded at $1 \times 10^{5}$ cells $/ \mathrm{mL}$, and grown until the density was approximately $4.7 \times 10^{6}$ and then incubated with amiodarone at a concentration of $9.7 \mathrm{nmol} / \mathrm{mL}$ for 2 hours. They were washed 3 times with ammonium formate $\mathrm{pH} 7.4$ by scrapping the cells off the flask with the first wash followed by 2 washes with centrifugation to avoid losing cells in the washing process. They were re-suspended in $10 \mathrm{~mL}$ of ammonium formate and the cell count was determined using a Vi-Cell XR (Beckman Coulter, USA). They were then pelleted and re-suspended in the appropriate volume of ProNet cell lysis buffer Tris pH7.6 (ESI Source Solution, LLC, Woburn, MA) depending on the cell density. Appropriate dilutions of the cell lysate were performed so the compound signal fell within the linear portion of the calibration curve and reserpine was used as an internal standard. The samples were analysed using a ABI4000 triple quadrupole (Sciex, Framingham, MA) coupled to a Waters UPLC system fitted with a Acquity UPLC BEH C18 $1.7 \mu \mathrm{m} 2.1 \times 50 \mathrm{~mm}$ coupled to a Acquity UPLC BEH C18 VanGuard cartridge 1.7um 2.1x5mm (Waters, Milford, MA). A gradient of 2 to $95 \%$ organic $(0.1 \%$ formic acid in acetonitrile, being the aqueous eluent $0.1 \%$ formic acid in water) in 5 minutes, operated at a flow rate of $0.5 \mathrm{~mL} / \mathrm{min}$ was employed.
Multiple reaction monitoring (MRM) methods were created for reserpine and amiodarone with at least 3 transitions for amiodarone. The amiodarone calibration curve was obtained using the same amount of cell lysate as the samples to account for any ion suppression ${ }^{39,40}$.

The assay validation showed very good sensitivity, precision and linearity with correlation efficiencies greater than 0.97 .

The amount of compound per cell was calculated by normalizing the LC-MS/MS result to the cell count obtained for each sample. Each sample was run 8 times and had 3 biological and 3 technical replicates, therefore 9 samples were analysed for each cell line, in a total of 72 LC-MS/MS runs. On average the number of cells analysed per injection was approximately 5000 cells. The data processing was done using Analyst (Sciex). The plots were generated in Statistica 64 (StatSoft).

Imaging Flow Cytometry. NR8383 cells were grown under the same conditions as described above for the LC-MS/MS experiment, but incubated with amiodarone for 72 hours. Hoescht 33342 (Thermo, USA) and Lysotracker Red DND-99 (Thermo, USA) were added so the final concentrations were $4 \mu \mathrm{g} / \mathrm{mL}$ for Hoescht and $75 \mu \mathrm{M}$ for Lysotracker red. The cells were then incubated for 30 minutes at $37^{\circ} \mathrm{C}$ and $5 \% \mathrm{CO}_{2}$, physically detached from the flask using a cell scrapper, washed 3 times and re-suspended in PBS (Gibco). They were subsequently sorted and analyzed using an ImageStream and IDEAS software (Amnis Corporation, USA).

Confocal fluorescent microscopy. NR8383 cells were grown and incubated following the same protocol as for the other imaging techniques. NR8383 cells were incubated with amiodarone for 72 hours at a concentration of $9.7 \mathrm{ug} / \mathrm{mL}$. Lysotracker red was added to the culture and incubated for 30 minutes. The media was removed and Fluoroshield ${ }^{\mathrm{TM}}$ with DAPI (Sigma-Aldrich, USA) mounting media was added to the slide and a cover slip placed on top. The cells were then imaged with a Leica SP5 confocal microscope using a 40x magnification lens.

High-throughput fluorescent microscopy. NR8383 cells were grown in a 96 well plate (Greiner) at the same relative density as previous experiments (T175 flasks and the Lab-Tek chamber slides) and incubated with amiodarone for 72 hours at a concentration of $9.7 \mathrm{nmol} / \mathrm{mL}$ using both Lysotracker red and Hoescht at the same concentration used for the flow cytometry experiment. They were imaged using an InCell 2000 (GE Heathcare, USA) with a $100 \mathrm{x}$ objective and 0.9 numerical aperture (NA) using fluorescent light; the instrument set up was done in accordance with the literature ${ }^{41}$.

At least 50 images were acquired per well and each concentration was run in duplicate, before and after freezedrying. The live images are not shown as focusing was difficult due to the semi-adherence nature of the NR8383 cell line. The experiment was therefore performed using a confocal microscope.

Electron Microscopy (EM). HeLa cells were grown as described above and pelleted and freeze-dried in the same manner as the samples prepared for ToF-SIMS. The samples were processed into Agar 100 epoxy resin (Agar Scientific, Stansted UK). The standard EM preparation involved manually processing the sample by dehydrating with a graded series of ethanol followed by immersion into acetone and final epoxy resin infiltration. 
The samples were then embedded and polymerised (at $60^{\circ} \mathrm{C}$ for 24 hours) and the resulting blocks were sectioned at approximately 80nm; stained with Uranyl Acetate/Lead Citrate. These were then examined in a Hitachi H7500 transmission electron microscope (TEM), operating at $80 \mathrm{kV}$ and representative digital micrographs taken.

\section{RESULTS AND DISCUSSION}

The four cell lines in this study, NR8383, HEK293, HepG2 and HeLa, were incubated using the same cell numbers with the same amiodarone concentrations for comparison by ToF-SIMS, LC-MS/MS and imaging flow cytometry. Using a blue nuclear Hoechst fluorescent dye and lysotracker red, the imaging flow cytometry images shown in Figure 1 illustrate the effect of amiodarone on the NR8383 cell line for doses up to 9.7 $\mathrm{nmol} / \mathrm{mL}$. It was apparent that the number as well as size of lysosomes increases with the amiodarone dose, also impacting overall cell size. The granularity of the cells or internal complexity is also affected by amiodarone dosing as can be seen by the side scatter light images in Figure 1. NR8383 cells are unable to metabolize amiodarone, which accumulates in the lysosomes and inhibits group XV phospholipase A2 $23,28,30$.

The effect of amiodarone on Hek293, HepG2 and HeLa cells is less well documented.

Since ToF-SIMS is a vacuum based technique, the samples were freeze dried for analysis; it is important to know the extent of cell damage or morphological changes caused during this process. We studied this using fluorescence microscopy imaging of the NR8383 cell line before and after freeze drying as shown in Fig 2. These data indicated that the cell morphology was not overly disrupted compared to live cells. In addition to the fluorescence microscopy data we also used electron microscopy to ensure freeze drying was not causing extensive damage of the ultra-structure of the cell. The EM shown in Fig 3 clearly demonstrates ultra-structure consistent with high quality preservation of the freeze-dried cells.

The electron microscopy results (Fig 3) indicated that around $95 \%$ of the cells looked viable with good ultra-structure including mitochondria with visible cristae as well as preserved Golgi complex, glycogen and endoplasmatic reticulum. Therefore, we can conclude that the freeze-drying process has little effect on the morphology of the cells which provides some confidence in the sample preparation method for the ToF-SIMS chemical imaging.

In Fig 4, we show 2D representations of the ToF-SIMS data that integrate the entire signal in the vertical direction as the sample is etched away using the gas cluster ion source (i.e. a cumulative plan view) for NR8383, HeLa, HeK293 and HepG2 cells. For each cell type, the large image to the left is a composite colour image with red representing the intensity of the ribose fragment $(\mathrm{m} / \mathrm{z} 81)$, blue representing the intensity of the phosphatidylcholine fragment $(\mathrm{m} / \mathrm{z}, 184)$ and green representing the amiodarone protonated molecular ion intensity $(\mathrm{m} / \mathrm{z}$ 646). These images are shown separately as a panel of small images on the right for clarity. The amiodarone is clearly detected for each cell type and the intensity appears higher in the HeLa and HepG2 cell lines. This high-resolution imaging mode ToF-SIMS data clearly shows compound and cell colocalization for all the cell lines at incubation concentration of $9.7 \mathrm{nmol} / \mathrm{mL}$ with 2 hours incubation. The relatively short incubation time, helps minimize the range in cell size in the NR8383 cell line that results from disruption of the lipid metabolism $^{33,42}$.

To generate sufficient individual cell measurements $(n>50)$ to compare with the average ensemble cell population measurements from LC-MS/MS, we use a low-resolution imaging mode to increase throughput since acquisition is faster. Figure S1 is the analogue of Fig 4 using this mode. It can be seen that the cells can generally still be resolved. For individual cell analysis, we define the perimeter of the cell by manually defining a region of interest perimeter on the acquired image and to calculate the integrated amiodarone ion intensity for the cell. In instances when the cells can't be resolved they are not included, and this occurs for approximately $10 \%$ of the cells analysed for all cell types. For individual cell analysis, we specify the perimeter of the cell by manually defining a region of interest perimeter on the acquired image and to calculate the integrated amiodarone ion intensity for the cell.

In Figure 5a we show a total of 329 individual cell ToF-SIMS measurements acquired in this way, of cell associated amiodarone for the four cell types (NR8383 $n=62$; HeLa $n=$ 86; Hek $293 n=57$; Hep G2 $n=124$ ). Each data point represents a single cell measurement, and reveal considerable cell-to-cell variation. In Figure S2, we plot frequency histograms show the distribution of drug accumulation. It can be seen that NR8383, Hek293 and HepG2 exhibit asymmetric distributions skewed with the modal amount significantly lower than the mean. This is in contrast to the HeLa cells. The variation between individual cells can have many reasons including that these cells are not synchronized within their cell cycles. This was not undertaken as the effect of the inhibitors required to achieve synchronization are unknown and could confuse the analysis; having cells within the culture in different parts of the cell cycle makes the model more biologically relevant.

Quantitative measurement achieved using LC-MS/MS of amiodarone concentration per cell from large populations $(>1$ million) are in Fig 5b. The LC-MS/MS shows that both HeLa and Hek293 cells have lower amiodarone uptake than the HepG2 and NR8383 cells. These cells are known to express less of the common ABC (ATP-Binding Cassette) and SLC (solute carrier) transporters ${ }^{30,43}$ and this might contribute to the lower amount of cell associated compound ${ }^{44,45}$. Detailed proteomics studies such as the one undertaken by Geiger, et $\mathrm{al}^{46}$ could be used to provide details on the differences in transporters between the different cell lines, but are beyond the scope of this study.

The higher drug uptake for the NR8383 cells can be explained by their biological function, as macrophages possess remarkable endocytosis capabilities ${ }^{47}$. Being an immune system cell they target microorganisms and other potential threats to the host. In this particular case, the threat is the compound and as a phagocyte they will try to internalize the compound to minimize the damage the drug could cause to other cells, with the intent to metabolize it or contain it within ${ }^{45,47}$. Since they cannot metabolize amiodarone, the internal drug concentration increases. Amiodarone disrupts the lipid metabolism in macrophages causing them to become enlarged with lipid and compound laden vesicles. The high amount of drug uptake in HepG2 cells is not surprising as amiodarone also causes phospholipidosis in these cells ${ }^{35}$. 
Most intracellular drug determination studies only report ratios of bound versus unbound compound ${ }^{21,34}$ so it is hard to draw a direct comparison between the intracellular drug concentration values obtained here and other studies. However, previous publications report intracellular drug concentration of the same order of magnitude as those presented in Fig $5 b^{48}$ measured by LC-MS/MS. Furthermore, the intracellular concentration also increases in the same order as the referenced study ${ }^{21,34,48}$ which is approximately 10 to 50 times higher than the extracellular media concentration ${ }^{49}$.

As one of the objectives of this paper was to determine if there was any correlation between the individual cell measurements by ToF-SIMS and the average cell population measurements with quantified LC-MS/MS, we compare these two data sources in Fig 6. The ToF-SIMS individual cell measurements for HeLa show a much higher intensity than would be expected based on the relationship of the other three cell types. Repeat measurements of the samples with the high-resolution imaging mode (Figure S3) are consistent with Figure 6, which indicates that the ToF-SIMS measurements are reproducible (between different days and different analysis modes). We do not have an explanation for why the HeLa data is inconsistent with the monotonic relationship between ToF-SIMS and LC-MS/MS for the other cells, and consequently we cannot in this study state with confidence that there is an overall correlation between amiodarone uptake in individual cell ToF-SIMS measurements and ensemble population LC-MS/MS measurements. The significant variability associated with the ToF-SIMS measurement is far greater than the reproducibility of the technique despite the relatively high number of cells analyzed indicating significant cell to cell drug uptake differences.

Elsewhere $^{50}$, we demonstrate that the SIMS protonated amiodarone molecular ion intensity is approximately linearly proportional to the amiodarone incubation concentration for NR8383 cells. The phenomena observed for HeLa cells, could be explained by the biological effect of amiodarone in HeLa cells at the concentration used for the study, which is relatively high and signs of cytotoxity are beginning to be observed. This could be addressed in the future using ToF-SIMS or using an orthogonal technique such as electron microscopy to investigate changes in lipid structures within HeLa cells with increasing levels of amiodarone.

\section{CONCLUSION}

We have shown that ToF-SIMS is able to identify and localize the drug amiodarone at therapeutic dosing concentrations (up to $9.7 \mathrm{nmol} / \mathrm{mL}$ ) in four different cell types, NR8383, HeLa, Hek293 and HepG2. A freeze-drying sample preparation method is used and a study with confocal microscopy incorporating nuclear and lysosome stains suggests that there is no significant disruption of the cell structure for the NR8383 cells (Figure 2). Equally, electron Microscopy of freeze dried HeLa suggests that the internal structural integrity of these cells is intact supporting the relevance of the ToF-SIMS of this cells prepared in this manner (Figure 3).

We show by ToF-SIMS that there is considerable variation in drug uptake at the individual cell level of the same type. Analysis of the frequency histograms of the amiodarone signal intensities reveals an asymmetric distribution for NR8383, HepG2 and Hek293 cells and a distribution closer to a normal distribution for HeLa cells (Figure S1). Quantitative measurements of the amount of cell associated amiodarone for the population average shows that NR8383 and HepG2 cells have the largest amount of amiodarone with on average 2.38 and $2.60 \mathrm{pg}$ per cell, respectively and HeLa and Hek293 have a significantly less amiodarone with 0.43 and $0.36 \mathrm{pg}$ per cell, respectively. For HeLa cells there is significant discrepancy between the single cell ToF-SIMS measurements compared with the LC-MS/MS measurement. For the other cells, a monotonic correlation could exist but the high scatter in the single cell data makes this difficult to state with confidence. Especially since we have no reasonable explanation to exclude the HeLa data.

We report a method using ToF-SIMS imaging that has good potential for studying the behavior of single cells and in the future to also look at drug efficacy and metabolism. This could open the possibility for further investigations of what causes cells to have such varied amounts of drug uptake. Challenges remain in the quantification of drug uptake.

\section{ACKNOWLEDGMENTS}

The authors thank Dr. Dave Hassall, Dr. Andy Nicholls, Dr. Mike Hann, Dr. Evie Malfoshie, Dr. Steve Hood, Dr. Simon Bate, Dr. Mike Lee, Dr. Bill Leavens and Dr. Gareth Wayne of GlaxoSmithKline and Dr. David Scurr from the University of Nottingham and Dr. Paulina Rakowska for discussions regarding this study. This work forms part of the "3D nanoSIMS" project in the Chemical and Biological programme of the National Measurement System of the UK Department of Business, Energy and Industrial strategy.

\section{AUTHOR INFORMATION}

\section{Corresponding Author}

*E-mail: carla.f.newman@gsk.com and ian.gilmore@npl.co.uk

\section{Funding Sources}

This work was funded by GSK.

Notes

The authors declare no competing financial interest. 


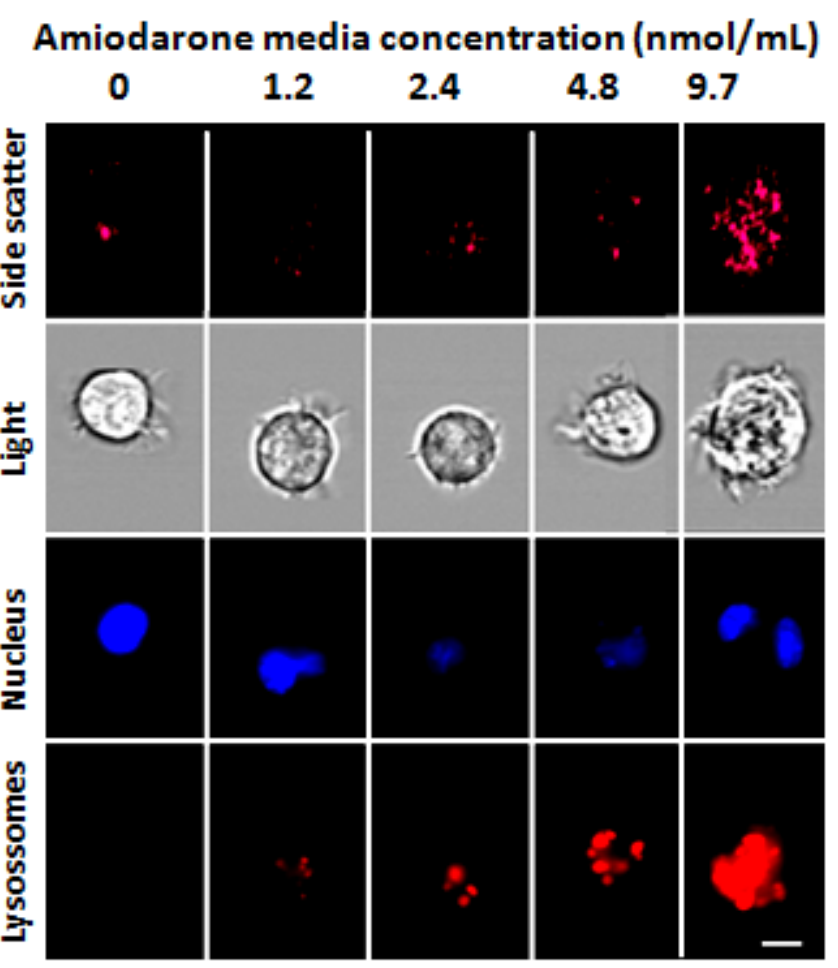

Figure 1: Imaging flow cytometry images of NR8383 cell line when incubated with different concentrations of amiodarone after 72 hours. The cells have been fluorescently stained with the nuclear dye Hoescht 33342 (blue) and Lysotracker (red), which stains the lysosome. The number and size of lysosomes increases with the treatment concentration (white line scale bar is equal to $10 \mu \mathrm{m}$ ). 

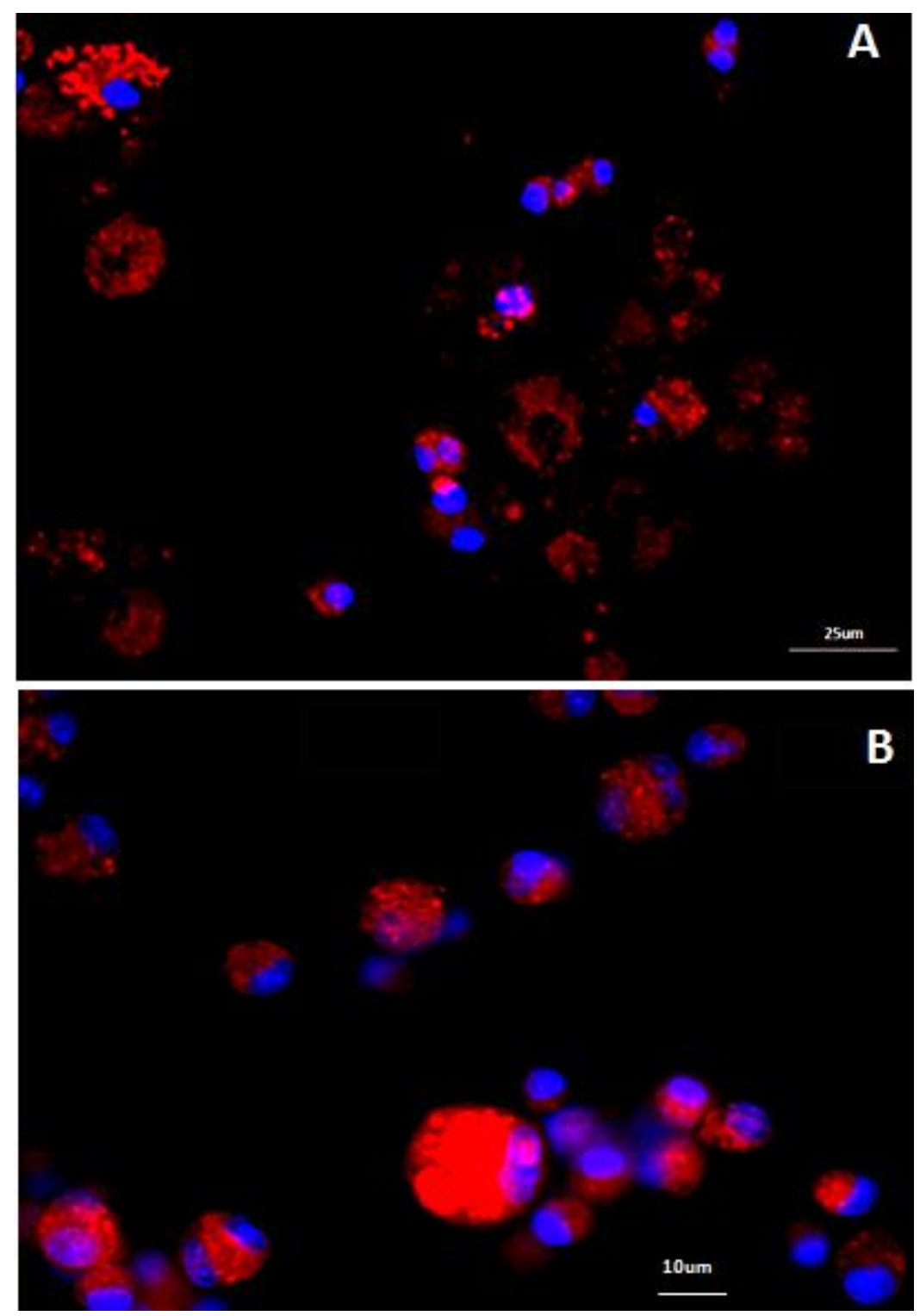

Figure 2: (A) Confocal fluorescence microcopy of live NR8383 cells incubated for 72 hours with $4.84 \mathrm{nmol} / \mathrm{mL}$ amiodarone exposure and staining as the live cells using a x40 objective on a Leica SP5 system with DAPI as a nuclear stain and Lysotracker red to stain the lysosome. (B) Image of high throughput fluorescent microcopy of freeze dried NR8383 cells undergoing the same amiodarone exposure and staining as the live cells using an Incell 2000 and a x100 objective. Both these experiments show NR8383 cells incubated for 72 hours in A the concentration is $4.84 \mathrm{nmol} / \mathrm{mL}$ amiodarone (as at $9.7 \mathrm{nmol} / \mathrm{mL}$ the vast majority of the cells were not adherent and therefore difficult to image) and in $\mathrm{B}$ is $9.7 \mathrm{nmol} / \mathrm{mL}$ amiodarone. In both $\mathrm{A}$ and $\mathrm{B}$ the highest possible spatial resolution images were acquired given the difference in cell state. 

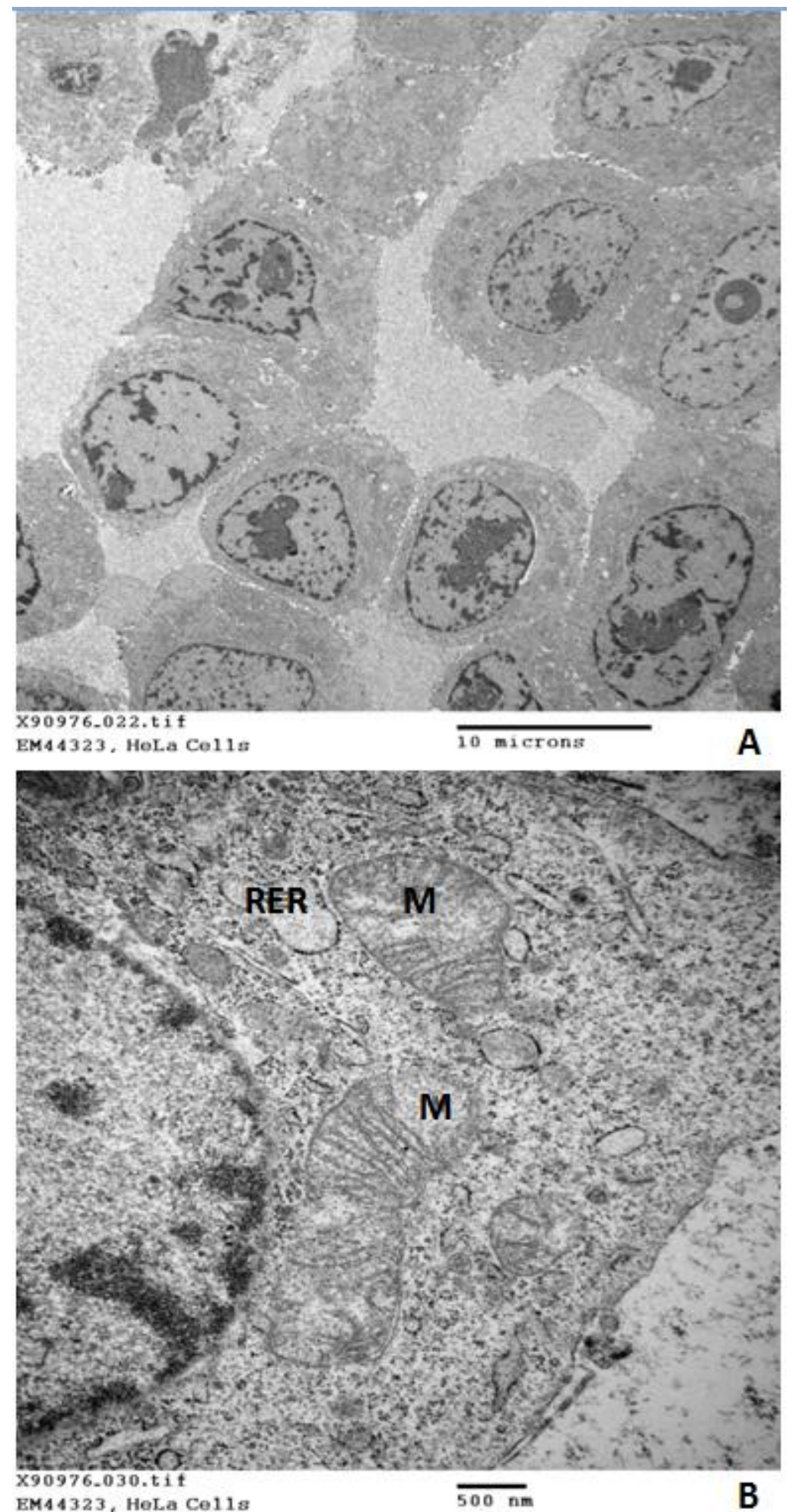

Figure 3: Electron microscopy images of freeze-dried HeLa cells. (A) HeLa cells section at direct magnification of 12000x. (B) HeLa cell mitochondria at 25000x direct magnification. RER stands for Rough endoplasmatic reticulum and M stands for mitochondria. 

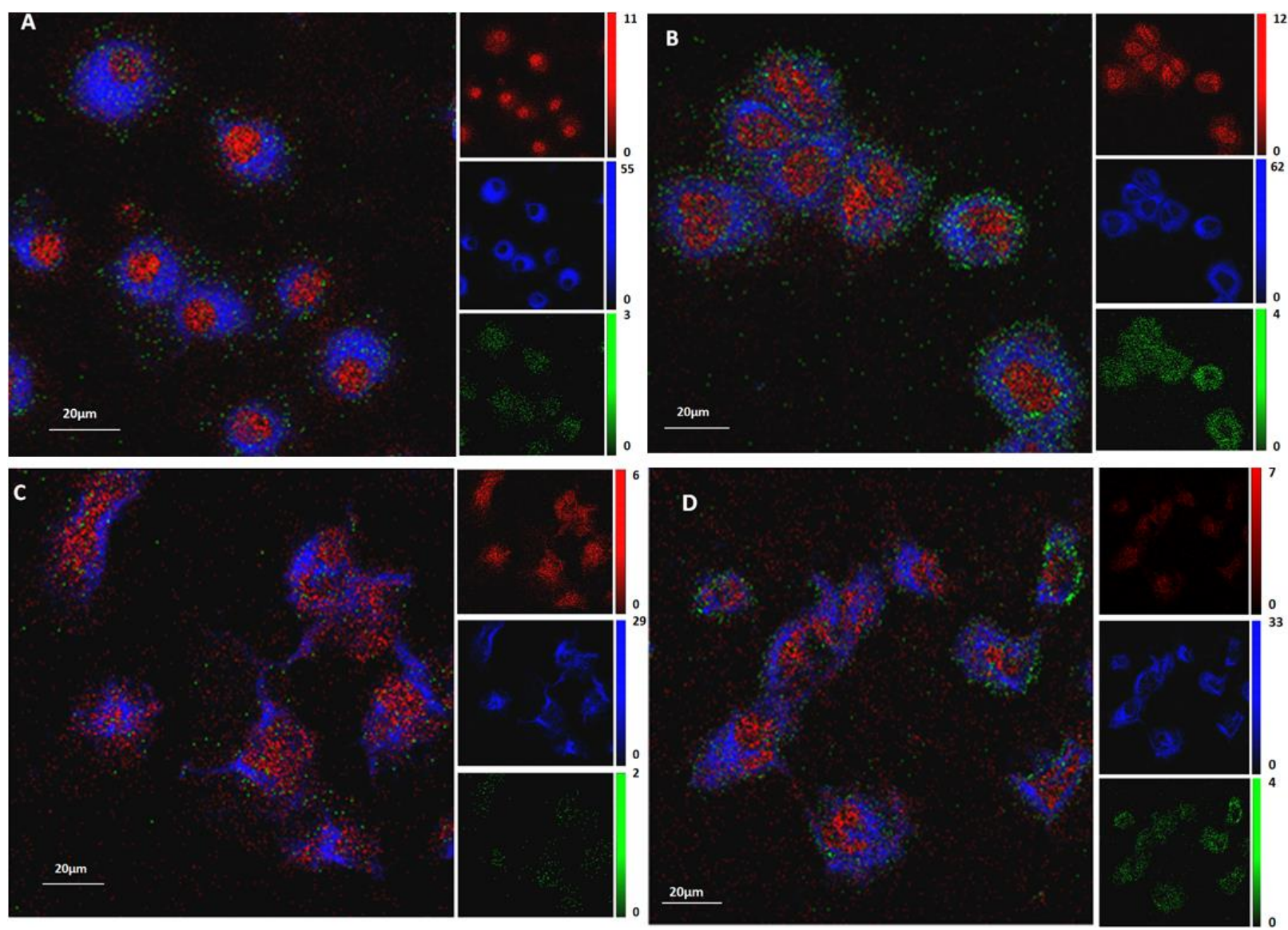

Figure 4 : ToF SIMS 2D images of a 3D data acquired from cells completely consumed by the argon cluster source during analysis. There were acquired in the higher spatial resolution mode, (A) NR8383 cells, (B) HeLa cells, (C) Hek 293 cells, (D) HepG2 cells, they were incubated for 2 hours with $9.7 \mathrm{nmol} / \mathrm{mL}$. Composite image where Red represents Ribose $\mathrm{m} / \mathrm{z} 81[\mathrm{M}+\mathrm{H}]^{+}$, blue shows the signal from the phosphatidylcholine lipid fragment $(\mathrm{m} / \mathrm{z}, 184))$, and green shows the amiodarone signal $(\mathrm{m} / \mathrm{z}, 646$ 

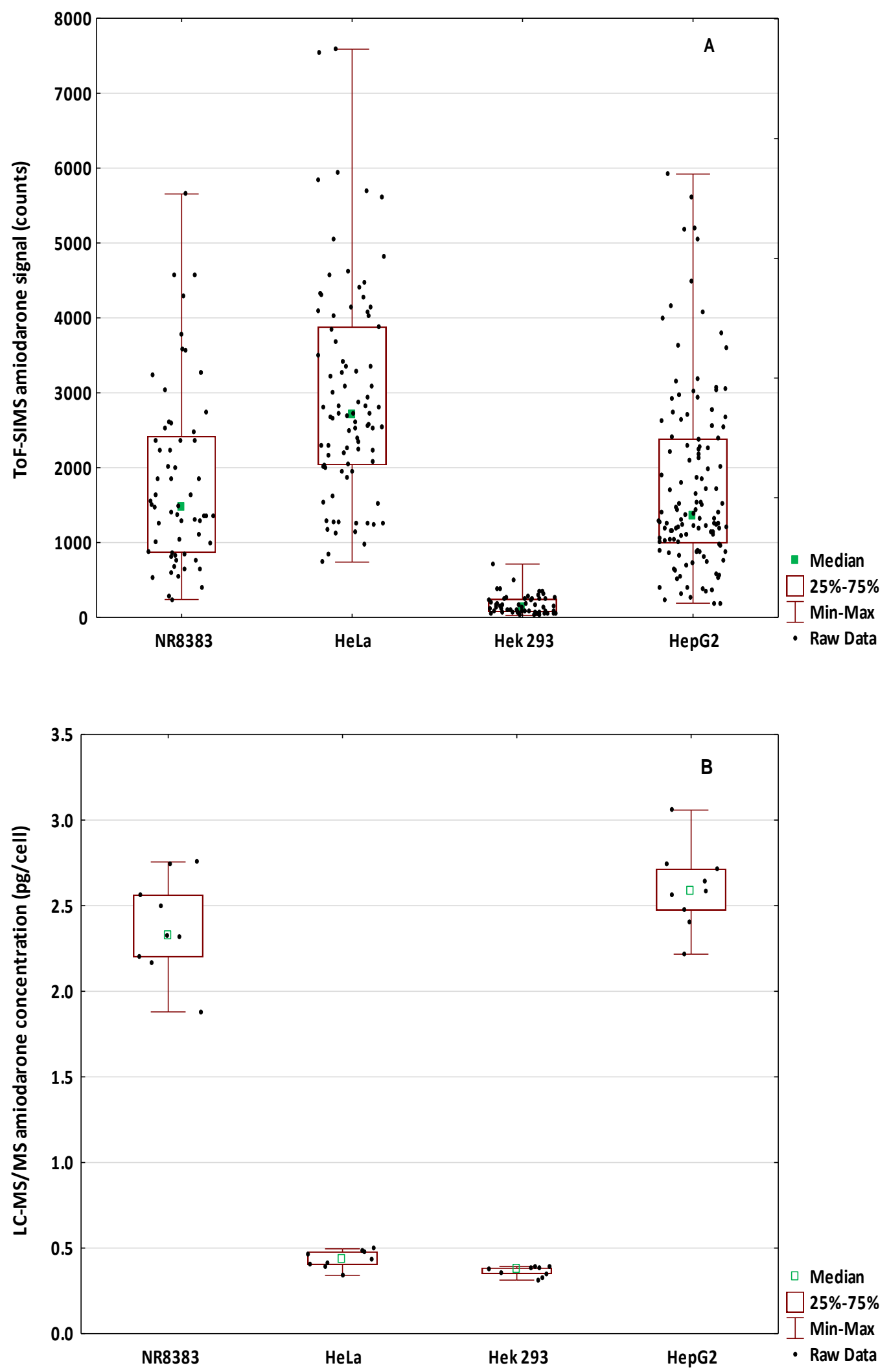

Figure 5: 5(A) box plot showing single cell population measurements for each cell type using the ToF-SIMS integrated amiodarone protonated molecular ion counts for each cell NR8383 $n=62$; HeLa $n=86$; Hek $293 n=57$; HepG2 $n=124$ (B) box plot showing cell population measurement using the LC-MS/MS assay, for each sample approximately $4.7 \times 10^{6}$ cells were used. 


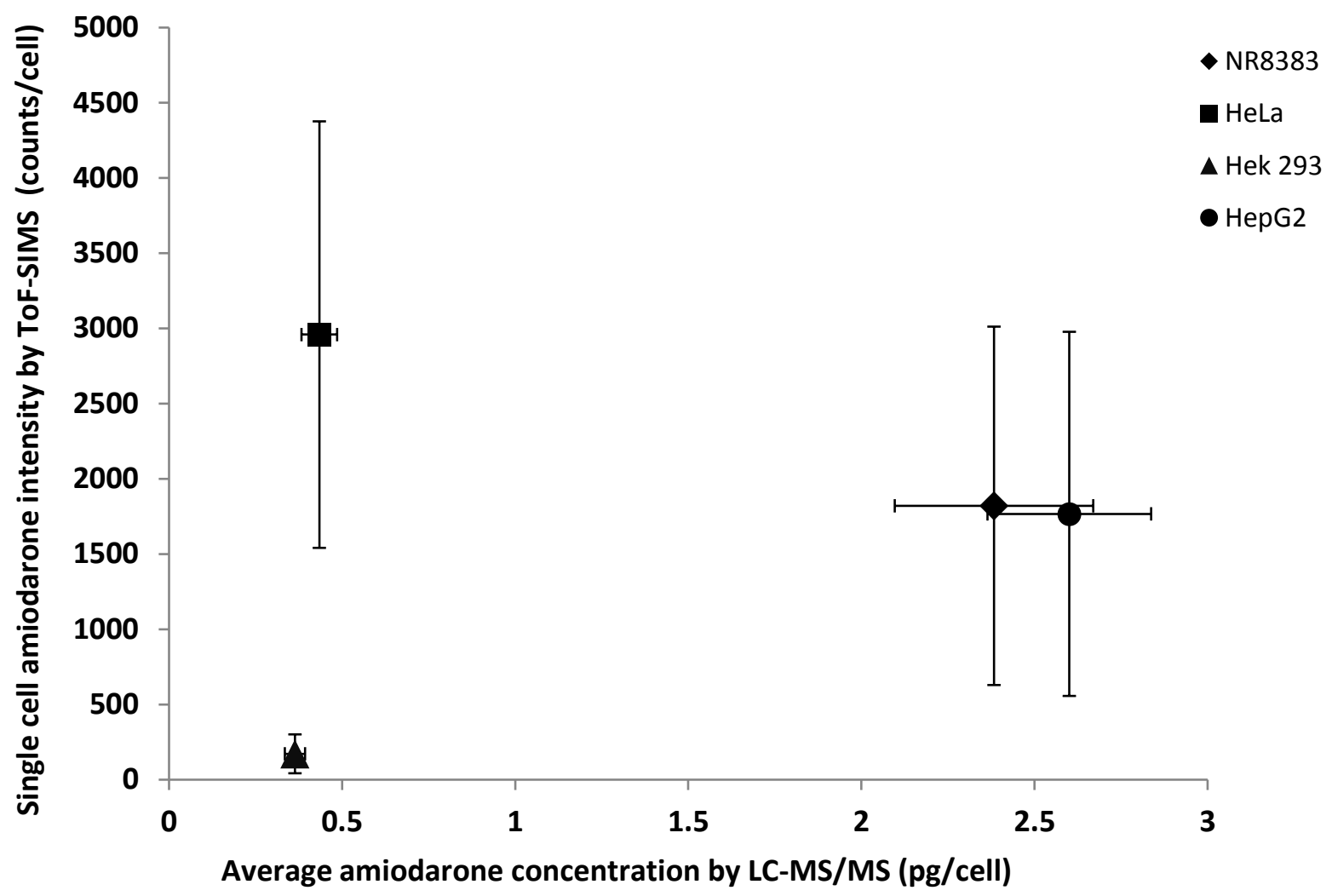

Figure 6: Comparison of the average cell associated amiodarone intensity determined by ToF-SIMS from a number of individual cells with the amiodarone cell uptake determine by LC-MS/MS from ensemble measurement of a large cell population (approximately 5000 per injection) for all cell lines incubated with $9.7 \mathrm{nmol} / \mathrm{mL}$ for 2 hours. The diamond represents the NR8383 cell line, the square represents HeLa cells, the circle represents HepG2 and the triangle represents Hek293 cells. The error bars represent one standard deviation from the average value. 


\section{REFERENCES}

1. Khanna, I. Drug discovery in pharmaceutical industry: Productivity challenges and trends. Drug Discov. Today 17, 1088-1102 (2012).

2. $\quad$ Dollery, C. T. Intracellular drug concentrations. Clin. Pharmacol. Ther. 93, 263-6 (2013).

3. $\quad$ Dollery, C. T. Lost in Translation (LiT). Br. J. Pharmacol. 171, 2269-2290 (2014).

4. Lee, J. A., Uhlik, M. T., Moxham, C. M., Tomandl, D. \& Sall, D. J. Modern Phenotypic Drug Discovery Is a Viable, Neoclassic Pharma Strategy. (2011).

5. Mitragotri, S., Burke, P. A. \& Langer, R. formulation and delivery strategies. Nat. Publ. Gr. 13, 655-672 (2014).

6. Chem, M. \& Hann, M. M. MedChemComm Molecular obesity, potency and other addictions in drug discovery. 2, (2011).

7. Hann, M. M. \& Keserü, G. M. in medicinal chemistry. 11, (2012).

8. Hopkins, A. L., Keserü, G. M., Leeson, P. D., Rees, D. C. \& Reynolds, C. H. The role of ligand efficiency metrics in drug discovery. Nat. Publ. Gr. 13, 105-121 (2014).

9. Hann, M. M. Molecular obesity, potency and other addictions in drug discovery. Multifaceted Roles Crystallogr. Mod. Drug Discov. 2, 183-196 (2015).

10. SINGLE- ANALYSES OF LIFE' S MOST. Nature 547, doi:10.1038/547019a (2017).

11. Spiller, D. G., Wood, C. D., Rand, D. A. \& White, M. R. H. Measurement of single-cell dynamics. 465, (2010).

12. Trim, P. J. et al. Spectrometry Imaging of Vinblastine in Whole Body Tissue Sections Matrix-Assisted Laser Desorption / Ionization-Ion Mobility Separation-Mass Spectrometry Imaging of Vinblastine in Whole Body Tissue Sections. Anal. Chem. 80, 8628-8634 (2008).

13. Zavalin A., Yang J., Haase A., Holle A., Caprioli R. , 61st Annual ASMS Conference on Mass Spectrometry and Allied Topics, 2014 At Minneapolis, M. NIH Public Access. 25, 1079-1082 (2015).

14. Rompp, A. Spengler, B. HistochemCellBio 2013, 139(6):756-783. Mass spectrometry imaging with high resolution in mass and space. 759-783 (2013). doi:10.1007/s00418-013-1097-6

15. Chéreau, R., Tønnesen, J. \& Nägerl, U. V. STED microscopy for nanoscale imaging in living brain slices. 88, 57-66 (2015).

16. Theillet, F. et al. Physicochemical Properties of Cells and Their E ff ects on Intrinsically Disordered Proteins ( IDPs ).

17. Yin, L. et al. How does fluorescent labeling affect the binding kinetics of proteins with intact cells? $412-416$ (2016). doi:10.1016/j.bios.2014.11.036. How

18. Hann, M. M., Leach, A. R. \& Harper, G. Molecular Complexity and Its Impact on the Probability of Finding Leads for Drug Discovery. 856-864 (2001).

19. Bemis, G. W. \& Murcko, M. A. The Properties of Known Drugs . 1 . Molecular Frameworks. 2623, 2887-2893 (1996).

20. Mateus, A., Matsson, P. \& Artursson, P. Rapid measurement of intracellular unbound drug concentrations. Mol. Pharm. 10, 2467-2478 (2013).

21. Passarelli, M. K. et al. Single-Cell Analysis: Visualizing Pharmaceutical and Metabolite Uptake in Cells with LabelFree 3D Mass Spectrometry Imaging. Anal. Chem. 87, 6696-6702 (2015).

22. Seki, S., Kobayashi, M., Itagaki, S., Hirano, T. \& Iseki, K. Biochimica et Biophysica Acta Contribution of organic anion transporting polypeptide OATP2B1 to amiodarone accumulation in lung epithelial cells. BBA - Biomembr. 1788, 911-917 (2009).

23. Ufuk, A., Somers, G., Houston, J. B. \& Galetin, A. In Vitro Assessment of Uptake and Lysosomal Sequestration of Respiratory Drugs in Alveolar Macrophage Cell Line NR8383. Pharm. Res. 32, 3937-3951 (2015).

24. Assmus, F. et al. In Vitro and in Silico Tools To Assess Extent of Cellular Uptake and Lysosomal Sequestration of Respiratory Drugs in Human Alveolar Macrophages. (2017). doi:10.1021/acs.molpharmaceut.6b00908

25. Med, J. P. R., Shamim, Z., Sarkar, P. R. \& Krishna, M. G. Journal of Pulmonary \& Respiratory Amiodarone Pulmonary Toxicity : A Case Report and Review of the Literature. 3-5 (2016). doi:10.4172/2161-105X.1000343

26. Habbab, K. M. \& Porter, S. R. Potential oral manifestations of cardiovascular drugs. 44, 769-773 (2010).

27. Kochiadakis, G. E. Efficacy and Safety of Oral Amiodarone in Controlling Heart Rate in Patients with Persistent Atrial Fibrillation who have Undergone. 336-340 (2005).

28. Antonini, J. M. ACCUMULATION OF AMIODARONE AND. 42, (1991).

29. Vorng, J.-L. et al. Semi-empirical rules to determine drug sensitivity and ionization efficiency in SIMS using a model tissue sample. Anal. Chem. (2016). doi:10.1021/acs.analchem.6b02894

30. Abe, A. \& Shayman, J. A. The role of negatively charged lipids in lysosomal phospholipase A2 function. J. Lipid Res. 50, 2027-2035 (2009).

31. Acton, Q. A. Issues in Biomedical Engineering Research and. (2013).

32. A.V.D Eb, USA FDA CTR for Biologicals Evaluation and Research Vaccines and Related Biological Products Advisory Committee Meeting, U. Haematoxylin, Mayer $\hat{a} €^{\mathrm{TM}} \mathrm{s}$ Hazardous according to criteria of Worksafe Australia. $2-4$ 
33. Gordon, L. J. et al. Direct Measurement of Intracellular Compound Concentration by RapidFire Mass Spectrometry Offers Insights into Cell Permeability. (2016). doi:10.1177/1087057115604141

34. Vickerman, J. \& Briggs, D. ToF-SIMS: Materials Analysis by Mass Spectrometry 2nd Edition. books.google.com (2013).

35. Nioi, P., Perry, B. K., Wang, E., Gu, Y. \& Snyder, R. D. In Vitro Detection of Drug-Induced Phospholipidosis Using Gene Expression and Fluorescent Phospholipid - Based Methodologies. 99, 162-173 (2007).

36. Brunelle, A., Touboul, D. \& Lapr, O. SPECIAL FEATURE : Biological tissue imaging with time-of-flight secondary ion mass spectrometry and cluster ion sources ION EMISSION UNDER ION AND CLUSTER. 985-999 (2005). doi:10.1002/jms. 902

37. Simon, G.M., Niphakis, M.J., Cravatt, B.F. NatChemBiol. 2013, 9, 200-2005. Determining target engagement in living systems. 9, 200-205 (2014).

38. Fletcher, J. S., Lockyer, N. P., Vaidyanathan, S. \& Vickerman, J. C. TOF-SIMS 3D biomolecular imaging of Xenopus laevis oocytes using buckminsterfullerene (C60) primary ions. Anal. Chem. 79, 2199-2206 (2007).

39. Bich, C. et al. Argon Cluster Ion Source Evaluation on Lipid Standards and Rat Brain Tissue Samples. (2013).

40. Grebe, S. K. G. \& Singh, R. J. LC-MS / MS in the Clinical Laboratory - Where to From Here ? 32, 5-31 (2011).

41. Healthcare, G. E. IN Cell Analyzer 2000. 28-31 (2000).

42. Buchser, W. et al. Assay Development Guidelines for Image-Based High Content Screening, High Content Analysis and High Content Imaging. 1-75 (2014).

43. Neve, E. P. A., Artursson, P., Ingelman-sundberg, M. \& Karlgren, M. An Integrated in Vitro Model for Simultaneous Assessment of Drug Uptake, Metabolism, and E ffl ux. (2013).

44. Mateus, A., Treyer, A., Wegler, C., Karlgren, M. \& Matsson, P. Intracellular drug bioavailability : a new predictor of system dependent drug disposition. Nat. Publ. Gr. 1-12 (2017). doi:10.1038/srep43047

45. Kell, D. B. \& Oliver, S. G. How drugs get into cells : tested and testable predictions to help discriminate between transporter-mediated uptake and lipoidal bilayer diffusion. 5, 1-32 (2014).

46. Geiger, T., Wehner, A., Schaab, C., Cox, J. \& Mann, M. Comparative Proteomic Analysis of Eleven Common Cell Lines Reveals Ubiquitous but Varying Expression of Most Proteins * $\square .1-11$ (2012). doi:10.1074/mcp.M111.014050

47. Mosser, D. M. \& Edwards, J. P. NIH Public Access. Genetics 8, 958-969 (2009).

48. Elick, H. E. S. \& Rove, J. R. U. G. MDCK (Madin - Darby Canine Kidney ) Cells : A Tool for Membrane Permeability Screening. 88, (1999).

49. Orlov, S. N., Platonova, A. A., Hamet, P. \& Grygorczyk, R. Cell volume and monovalent ion transporters : their role in cell death machinery triggering and progression. (2013). doi:10.1152/ajpcell.00040.2013

50. Melissa K.Passarelli, Alexander Pirkl, Rudolf Moellers, Dmitry Grinfeld, Felix Kollmer, Rasmus Havelund, Carla F. Newman, Peter S. Marshall, Henrik Arlinghaus, Morgan R. Alexander, Andy West, Stevan Horning, Ewald Niehuis, Alexander Makarov, C. T. D. and I. S. G. The 3D OrbiSIMS - A New Method for Label-Free Metabolic Imaging with Sub-cellular Lateral Resolution and High Mass Resolving Power. Submited (2017). 
